\title{
Research Article \\ Efficient Option Pricing in Crisis Based on Dynamic Elasticity of Variance Model
}

\author{
Congyin Fan, Kaili Xiang, and Peimin Chen \\ School of Economics and Mathematics, Southwestern University of Finance and Economics, Chengdu, Sichuan 611130, China \\ Correspondence should be addressed to Peimin Chen; chenpeimin@swufe.edu.cn
}

Received 11 December 2015; Accepted 8 March 2016

Academic Editor: Leonid Shaikhet

Copyright (C) 2016 Congyin Fan et al. This is an open access article distributed under the Creative Commons Attribution License, which permits unrestricted use, distribution, and reproduction in any medium, provided the original work is properly cited.

\begin{abstract}
Market crashes often appear in daily trading activities and such instantaneous occurring events would affect the stock prices greatly. In an unstable market, the volatility of financial assets changes sharply, which leads to the fact that classical option pricing models with constant volatility coefficient, even stochastic volatility term, are not accurate. To overcome this problem, in this paper we put forward a dynamic elasticity of variance (DEV) model by extending the classical constant elasticity of variance (CEV) model. Further, the partial differential equation (PDE) for the prices of European call option is derived by using risk neutral pricing principle and the numerical solution of the PDE is calculated by the Crank-Nicolson scheme. In addition, Kalman filtering method is employed to estimate the volatility term of our model. Our main finding is that the prices of European call option under our model are more accurate than those calculated by Black-Scholes model and CEV model in financial crashes.
\end{abstract}

\section{Introduction}

Nowadays, more and more researchers focus on stock option pricing problems. In different environments, such as bull markets or bear markets, the returns of stock prices have different properties and distributions to follow, based on which many different models (see [1-7]) are proposed and some analytic formulae or approximations are provided. For some classical models without analytic solutions, lots of efficient numerical methods (see [8-14]) are presented in detail. In addition, by employing some existing model and numerical methods, some researches (see $[15,16]$ ) focus on the empirical tests on actual data, and some great findings are observed in the real derivative markets.

In recent two decades, the events of financial crisis, such as the stock market crash in 1987 and the subprime crisis in 2008, show that extreme events have great effects on financial markets and cannot be negligible in option pricing problems. So, more and more researches focus on the dynamic markets that experience sharp crashes, and lots of contributions on option pricing models are presented. For instance, from an empirical view the dynamic financial markets following the occurrence of a financial crash have been studied in $[17,18]$.
In 2003, Sornette in [19] finds that the postcrash stock prices follow a converging oscillatory motion through a nonparameter method. In addition, Lillo and Mantenga in [18] show that ex-postfinancial markets of crisis have characteristics of a power-law relaxation decay. By using results of [19], ElKhatib et al. in [20] study European option pricing model with postcrash relaxation times in 2007. In [7], Zhu and Galbraith present an evidence that stock returns can be fitted by Student's $t$-distribution during postcrash relaxation times, which is consistent with the results in [17, 18]. In 2011, Markose and Alentorn in [6] employ the generalized extreme value distribution to model the implied risk neutral density function and provide a flexible framework that captures the negative skewness and excess kurtosis of returns in turbulent financial markets.

If markets are in heavy crisis, the volatility of financial assets changes sharply and is much bigger than that in bull markets. To grasp unstable volatility, many kinds of stochastic volatility models, such as Heston model, Hull-White model, GARCH model, and CEV model, are proposed and option pricing formula under risk neutral measure is given or approximated. In these models, the volatility terms are constructed by parametric processes, such as Cox-Ingersoll-Ross (CIR) 
process or Ornstein-Uhlenbeck (OU) process, and so forth, of which the parameters are assumed to be constants. Further, Yoon et al. in $[21,22]$ study problems of option pricing under stochastic elasticity of variance model and their works enhance the existing option price structures in view of flexibility and applicability through the market prices of elasticity risk. For these processes, there exist some certain distributions they followed. But, in the real stock markets, crashes have different shapes and properties without any fixed rules to obey. Moreover, lots of extreme values of returns accumulate on tails of return distributions. These facts lead to the point that it is impossible to use a certain distribution or process to describe the information hidden in volatility term. In this paper we put forward a semiparametric model with parametric drift coefficient and nonparametric volatility term to imitate the motion of stock prices in crashes. In this model, the volatility term is a compound function based on stock prices to the power of a nonparametric function, which implies that the model has dynamic elasticity of variance. Our model is an extension of a constant elasticity of variance (CEV) model (see [4]), in which the power of the volatility term is a constant in response to observations of an inverse relationship between prices and volatility of prices under some conditions. In this paper, we name our model as dynamic elasticity of volatility (DEV) model.

Based on the DEV model, under risk neutral measure the partial differential equation (PDE) for the prices of European call option is derived. Then, Crank-Nicolson method, which is unconditionally stable, is used on this PDE to simulate option prices. Further, the Kalman filtering method and least square method are employed to fit the volatility part which exhibits exponentially decaying oscillatory movement. For comparison, we concurrently estimate parameters of the DEV model, BS model, and CEV model by the same samples to obtain the corresponding option prices. Our main contributions and findings in this paper include that we first put forward a dynamic elasticity of variance model to explain the big changes of volatility and of option prices in crashes. Moreover, in empirical test European call option prices are shown to be approached more accurately by DEV model than BS model and CEV model, especially in the ten percent interval nearby the point of "at-the-money." In the area far from this point, the solutions of DEV model and CEV model are very similar, almost the same. This finding guides us to the fact that in the real trading activity we can depend on DEV model with confidence to price European options in the surroundings of "at-the-money."

The remainder of this paper is arranged as follows. In Section 2, we put forward our model and derive the PDE satisfied by European call option by using the risk neutral principle. In Section 3, Kalman filtering method and the nonlinear least square method are employed to determine the nonparametric term and some parameters. In Section 4, we take three samples with respect to the historical daily data of closed stock prices of S\&P500 index to estimate parameters of DEV, CEV, and BS models, respectively. And some numerical results of option prices and their analysis are presented in Section 5. And then in the last section we make some conclusions.

\section{Pricing Model}

In this section, first we put forward a new model, dynamic elasticity of variance (DEV) model, by extending the CEV model to fit the process of stock prices in crashes. Then, based on this model, the partial differential equation (PDE) satisfied by European option prices is derived and verified.

2.1. Dynamic Elasticity of Variance Model. Let $W_{t}, t \in[0, T]$, be a Brownian motion on a probability space $(\Omega, \mathscr{F}, P)$ and let $\mathscr{F}, t \in[0, T]$ be a filtration generated by $W_{t}$. First, let us recall the classical CEV model in [4], which follows a stochastic process as

$$
d S_{t}=\mu S_{t} d t+\delta S_{t}^{\theta / 2} d W_{t}
$$

where $(\mu, \delta, \theta)$ are constant parameters and $S_{t}$ is the stock price at time $t$. At the events of crashes, although some small sizes of rebounding follow, generally the stock prices would be sharply decreasing, which leads to the fact that the volatility of stock prices becomes increasing greatly. But for the CEV model, usually $\theta>2$, then the volatility term $\delta S_{t}^{\theta / 2-1}$ has the same monotonic trend as the stock prices $S_{t}$ (see [23]). This is a contradiction. Therefore, it is necessary for us to improve the classical CEV model to be an efficient one to reflect the inverse relationship between stock prices and their volatility in crashes. On the other hand, in crashes the volatility changes turbulently in short period and it is hard to find an effective process or distribution to express this phenomenon. In this section, to overcome these difficulties we extend the constant parameter $\theta$ to be a dynamic function $f(t)$ and propose a dynamic elasticity of variance model as follows:

$$
d S_{t}=\mu S_{t} d t+\delta S_{t}^{f(t) / 2} d W_{t}
$$

where $\mu$ and $\delta$ are constant parameters as those in CEV model. If $f(t)$ is a constant, then it degenerates to the classical CEV model.

2.2. Pricing European Call Option. In this part, the price of European call option is considered as a contingent claim that pays $\max \left\{S_{T}-K, 0\right\}$ at maturity $T$, and $K$ is the strike price.

Let $\Theta(u)=(\mu-r) / \delta S_{u}^{f(u) / 2-1}$ be a real valued predictable process, $B_{t}$ a process defined by

$$
B_{t}=\int_{0}^{t} \Theta(u) d u+W_{t}
$$

and $\rho_{t}$ a function of time $t$, which is given by

$$
\rho_{t}=\exp \left(\int_{0}^{t} \Theta(u) d W_{u}-\frac{1}{2} \int_{0}^{t} \Theta^{2}(u) d u\right) .
$$

Define a new probability measure $Q$ by

$$
Q(A)=\int_{A} \rho_{t}(\omega) d P(\omega), \quad \forall A \in \mathscr{F} .
$$


Then we can check that $E\left[\rho_{T}\right]=1$ and $E\left[\int_{0}^{t} \Theta^{2}(u) \rho_{u}^{2} d u\right]<\infty$. Moreover, by Girsanov theorem, $B_{t}$ is also a Brownian motion under the measure $Q$. Consequently,

$$
\begin{aligned}
d S_{t} & =\mu S_{t} d t+\delta S_{t}^{f(t) / 2} d W_{t} \\
& =\mu S_{t} d t+\delta S_{t}^{f(t) / 2}\left[d B_{t}-d \int_{0}^{t} \frac{\mu-r}{\delta S_{u}^{f(u) / 2-1}} d u\right] \\
& =r S_{t} d t+\delta S_{t}^{f(t) / 2} d B_{t} .
\end{aligned}
$$

Suppose that the market is complete and arbitrage-free. Now, let $C\left(t, S_{t}\right)$ be the European option price at time $t$. We replicate this option by a portfolio constructed by $\Delta(t)$ shares of stocks and money with amount of $C\left(t, S_{t}\right)-\Delta(t) S_{t}$, which is a risk-free asset. Let $\Pi_{t}=C\left(t, S_{t}\right)-\Delta(t) S_{t}$; then $d \Pi_{t}=r \Pi_{t} d t$. By Itô formula, from (6) we can obtain

$$
\begin{aligned}
d \Pi_{t}= & d C\left(t, S_{t}\right)-\Delta(t) d S_{t} \\
= & \frac{\partial C}{\partial t} d t+\frac{\partial C}{\partial S_{t}} d S_{t}+\frac{1}{2} \frac{\partial^{2} C}{\partial S_{t}^{2}} d S_{t} d S_{t}-\Delta(t) d S_{t} \\
= & \frac{\partial C}{\partial t} d t+\frac{\partial C}{\partial S_{t}}\left[r S_{t} d t+\delta S^{(1 / 2) f(t)} d B_{t}\right] \\
& +\frac{1}{2} \delta^{2} S_{t}^{f(t)} \frac{\partial^{2} C}{\partial S_{t}^{2}} d t \\
& -\Delta(t)\left[r S_{t} d t+\delta S_{t}^{(1 / 2) f(t)} d B_{t}\right] \\
= & {\left[\frac{\partial C}{\partial t}+r S_{t} \frac{\partial C}{\partial S_{t}}+\frac{1}{2} \delta^{2} S_{t}^{f(t)} \frac{\partial^{2} C}{\partial S_{t}^{2}}-\Delta(t) r S_{t}\right] d t } \\
& +\left[\frac{\partial C}{\partial S_{t}} \delta S_{t}^{(1 / 2) f(t)}-\Delta(t) \delta S_{t}^{(1 / 2) f(t)}\right] d W_{t} \\
= & r \Pi_{t} d t=r\left(C-\Delta(t) S_{t}\right) d t .
\end{aligned}
$$

In (7), choose $\Delta(t)=\partial C / \partial S_{t}$; then the partial differential equation satisfied by European call option is obtained as follows:

$$
\frac{\partial C}{\partial t}+r S_{t} \frac{\partial C}{\partial S_{t}}+\frac{1}{2} \delta^{2} S_{t}^{f(t)} \frac{\partial^{2} C}{\partial S_{t}^{2}}-r C=0
$$

Moreover, at the expiry date $T$ the boundary condition is given by

$$
C\left(T, S_{T}\right)=\max \left(S_{T}-K, 0\right) .
$$

In (8), generally $f(t)$ is not zero and the equation is a nonlinear partial differential equation. So, it is hard and sometimes impossible to write out the explicit form of the solution of (8) and (9). In this paper, we use a numerical method, finite difference method, to simulate the solution.

\section{Parameter Estimation of DEV Model}

During the postcrash relaxation times, it is well known that the stock market is turbulent and it needs a period of time to recover stability. So, in such a case, the CEV model cannot capture the real features in that the elasticity of volatility with respect to stock prices is a constant. For this event, we should find a function or process with features of convergence and power-law relaxation decay to replace constant elasticity of volatility term. Consequently, a candidate function $f(t)=a+$ $A e^{b t} \sin (\omega t)$ is considered and the parameters of $f(t)$ will be determined in the following part.

To get the parameters $(a, A, b, \omega)$ of $f(t)$, the method in [23] is employed as follows. For the process

$$
d S_{t}=\mu\left(t, S_{t}\right) d t+\sigma\left(t, S_{t}\right) d W_{t},
$$

the volatility term $\sigma^{2}\left(t, S_{t}\right)$ can be estimated by $E\left[V_{t} \mid S_{t}\right]$ as

$$
E\left[V_{t} \mid S_{t}\right] \longrightarrow \sigma^{2}\left(t, S_{t}\right), \quad \Delta t \longrightarrow 0,
$$

where

$$
V_{t}=\frac{2}{\alpha \Delta t}\left[\frac{S_{t+\Delta t}^{1+\alpha}-S_{t}^{1+\alpha}}{(1+\alpha) S_{t}^{1+\alpha}}-\frac{S_{t+\Delta t}-S_{t}}{S_{t}}\right],
$$

where $\alpha$ is a constant and $\Delta t$ is the step size of time interval. It also shows that the conditional variance $\operatorname{Var}\left(V_{t} \mid S_{t}\right)$ is minimized when

$$
\alpha=-\frac{13}{11}-\frac{12}{11} \frac{\mu}{E\left[V_{t} \mid S_{t}\right]} .
$$

Computationally, we start with an initial value of $\alpha$ and then obtain a value of $V_{t}$ from (12). The parameter $\mu$ can be easily estimated by using the mean of sample returns and denoted by $\widehat{\mu}$. Replacing $\mu$ by $\widehat{\mu}$ in (13), then a renewed value of $\alpha$ is yielded. Further, we iterate these two steps until the last two values of $\alpha$ are within a certain error tolerance. In the end, we replace $\sigma^{2}\left(t, S_{t}\right)$ by $V_{t}$.

Using the method above, $\delta^{2} S_{t}^{f(t)}$ in the model (2) can be estimated by $V_{t}$; namely,

$$
V_{t}=\delta^{2} S_{t}^{f(t)}
$$

Consequently,

$$
\ln V_{t}=\ln \delta^{2}+f(t) \ln S_{t} .
$$

Let $y_{t}=\ln V_{t}, \alpha_{t}=\ln \delta^{2}, \beta_{t}=f(t)$, and $x_{t}=\ln S_{t}$; then (15) can be estimated by the following regression equation:

$$
\begin{aligned}
y_{t} & =\alpha_{t}+\beta_{t} x_{t}+e_{t}, \quad e_{t} \sim N\left(0, \delta_{e}^{2}\right), \\
\alpha_{t+1} & =\alpha_{t}+\eta_{t}, \quad \eta_{t} \sim N\left(0, \delta_{\eta}^{2}\right), \\
\beta_{t+1} & =\beta_{t}+\varepsilon_{t}, \quad \varepsilon_{t} \sim N\left(0, \delta_{\varepsilon}^{2}\right) .
\end{aligned}
$$

Equations (16)-(18) construct a stable space model. If we take $A_{t}=\left[\alpha_{t}, \beta_{t}\right]^{\tau}$, then (16)-(18) can be rewritten as

$$
\begin{aligned}
y_{t} & =\left[1, x_{t}\right] A_{t}+e_{t}, \\
A_{t+1} & =\left(\begin{array}{ll}
1 & 0 \\
0 & 1
\end{array}\right) A_{t}+\left(\begin{array}{c}
\eta_{t} \\
\varepsilon_{t}
\end{array}\right) .
\end{aligned}
$$


Using Kalman filtering method for equations (19), then Kalman filtering algorithm can be written as follows:

$$
\begin{aligned}
v_{t} & =y_{t}-\left[1, x_{t}\right] A_{t \mid t-1}, \\
\Lambda_{t} & =\left[1, x_{t}\right] \Sigma_{t \mid t-1}\left[1, x_{t}\right]^{\tau}+\delta_{\varepsilon}^{2}, \\
K_{t} & =\Sigma_{t \mid t-1}\left[1, x_{t}\right]^{\tau} \Lambda_{t}^{\tau}, \\
L_{t} & =I-K_{t}\left[1, x_{t}\right], \\
A_{t+1 \mid t} & =A_{t \mid t-1}+K_{t} \nu_{t}, \\
\Sigma_{t+1 \mid t}= & \Sigma_{t+1 \mid t} L_{t}^{\tau}+\left(\begin{array}{cc}
\delta_{\eta}^{2} & 0 \\
0 & \delta_{\varepsilon}^{2}
\end{array}\right), \\
\max \ln \left[L\left(\delta_{\varepsilon}, \delta_{\eta}\right)\right]= & -\frac{T}{2} \ln (2 \pi) \\
& -\frac{1}{2} \sum_{t=1}^{T}\left[\ln \left(\Lambda_{t}\right)+\frac{v_{t}^{2}}{\Lambda_{t}}\right] .
\end{aligned}
$$

So, according to (20), by using two given initial values $A_{1 \mid 0}$ and $\Sigma_{1 \mid 0}$, the values of $A_{t}$ can be calculated. Therefore, we obtain the estimated values of $\ln \widehat{\delta}^{2}$ and $\hat{f}(t)$.

What is more, to determine the parameters $\phi=$ $(a, b, A, \omega)$ of $f(t)$, the nonlinear least square method is employed as follows:

$$
\min _{\phi}\|f(t)-\widehat{f}(t)\|^{2}
$$

\section{Empirical Parameter Estimations}

In this section, first we download three data fragments with respect to historical daily data of closed prices of S\&P500 index from the website http://finance.yahoo.com/ as our selected samples. The time interval of one covers the period from October 17, 1997, to October 17, 1998, during which the Asian crisis happened and the stock markets of America were infected heavily. Another sample is collected from September 10,2001 , to September 10, 2002, in which the " $9 / 11$ " event took place and influenced the stock markets greatly. The period of the last sample is from September 16, 2008, to September 15, 2009 , in which the big event of subprime crisis occurred and stock markets endured deep crashes.

4.1. Empirical Parameter Estimation of DEV Model. In this subsection, We employ Kalman filtering method of last section to obtain the values of $f(t)$ and $\delta$, respectively, in model (2) and further to estimate the parameters of $f(t)$. In addition, BS model and CEV model are estimated on the same samples for comparison and analysis.

For (11)-(13), the certain error tolerance we take is 0.005 . In fact, taking a smaller error tolerance, however, does not change the numerical results significantly in our tests. Hence, for different samples, taking 0.005 as the error tolerance is sufficient to obtain effective results with enough precision. Inputting an initial value of $\alpha$ into (12), then, by the iteration algorithm provided in Section 3, values of $V_{t}$ can be obtained

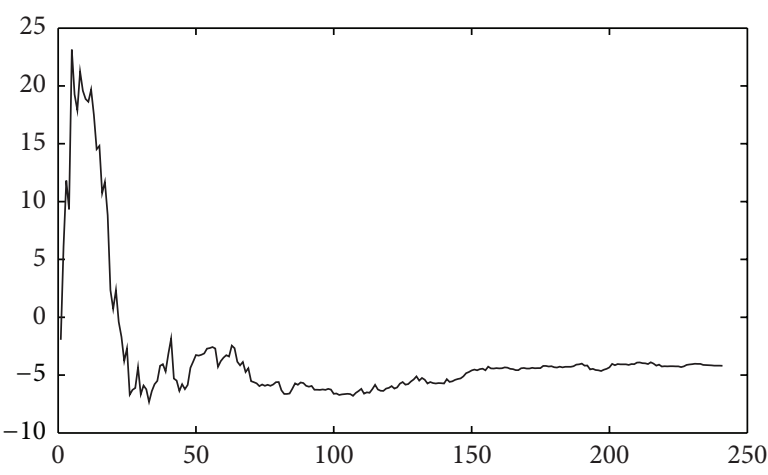

FIgURE 1: Values of $f(t)$ during Asian crisis.

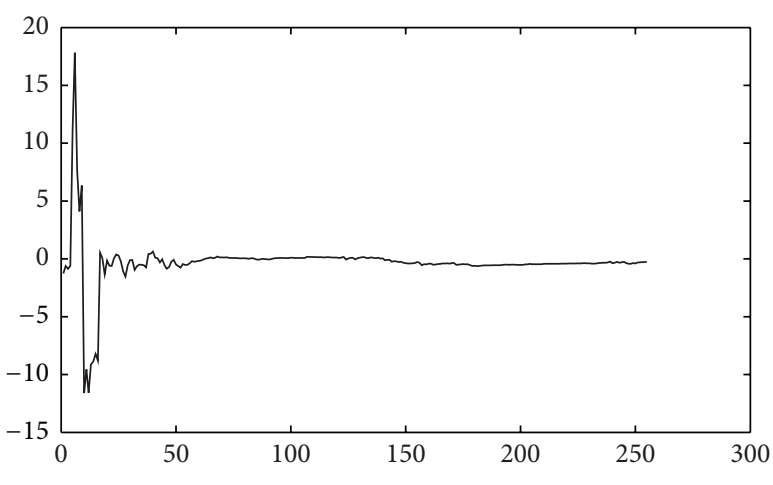

Figure 2: Values of $f(t)$ during “9/11" event.

under reasonable convergence. Based on the achieved results of $V_{t}$, for given initial values

$$
\begin{aligned}
& A_{1 \mid 0}=\left(\begin{array}{l}
1 \\
1
\end{array}\right), \\
& \Sigma_{1 \mid 0}=\left(\begin{array}{cc}
500 & 0 \\
0 & 500
\end{array}\right),
\end{aligned}
$$

then by Kalman filtering method the value of $\delta$ can be obtained in Table 1, and the scatter diagrams of $f(t)$ for three different samples are represented in Figures 1-3, which are used as real values of $f(t)$. Now, to determine the coefficients of $f(t)$, the minimum criteria (21) are used, and then parameters in $f(t)$ are obtained, which are also exhibited in Table 1.

The real values of $f(t)$ and estimated values $\hat{f}(t)$ are displayed in Figures $4-6$, in which the real line and dotted line represent real values and estimated values, respectively.

4.2. Empirical Parameter Estimation of CEV Model. In CEV model, to obtain point estimators of $(\theta, \delta)$, firstly we find an estimation of $\sigma_{t}^{2}$, which satisfies $\sigma_{t}^{2}=\delta^{2} S_{t}^{\theta}$. In fact, for the same samples, $\sigma_{t}^{2}$ can be approached by $E\left[V_{t} \mid S_{t}\right]$ as $\Delta t \rightarrow 0$ from (11)-(13) and that the conditional variance $\operatorname{Var}\left[V_{t} \mid S_{t}\right]$ is minimized when (13) holds. 
TABLE 1: Summary of parameter estimations.

\begin{tabular}{|c|c|c|c|c|}
\hline \multicolumn{2}{|c|}{ Event } & Asian crisis & “9/11" event & Subprime crisis \\
\hline \multicolumn{2}{|c|}{ Data } & Oct. 17, 1997-Jun. 16, 1998 & Sep. 10, 2001-Jun. 15, 2002 & Sep. 16, 2008-Jun. 30, 2009 \\
\hline \multirow{2}{*}{ BS } & $\mu$ & 0.1814 & 0.1309 & 0.2014 \\
\hline & $\sigma$ & 0.1130 & 0.2300 & 0.1853 \\
\hline \multirow{3}{*}{ CEV } & $\mu$ & 0.1814 & 0.1309 & 0.2014 \\
\hline & $\delta$ & 9.8322 & 9.1771 & 9.6514 \\
\hline & $\theta$ & -1.8500 & -2.0131 & -1.9831 \\
\hline \multirow{6}{*}{ DEV } & $\mu$ & 0.1814 & 0.1309 & 0.2014 \\
\hline & $\delta$ & 1.7932 & 2.0861 & 1.4730 \\
\hline & $\alpha$ & -4.2010 & -0.2832 & -3.2581 \\
\hline & $A$ & 75.0210 & 27.4763 & 70.0012 \\
\hline & $b$ & -0.1651 & -0.8690 & -39.9924 \\
\hline & $\omega$ & -0.1520 & 0.3501 & 0.1508 \\
\hline
\end{tabular}

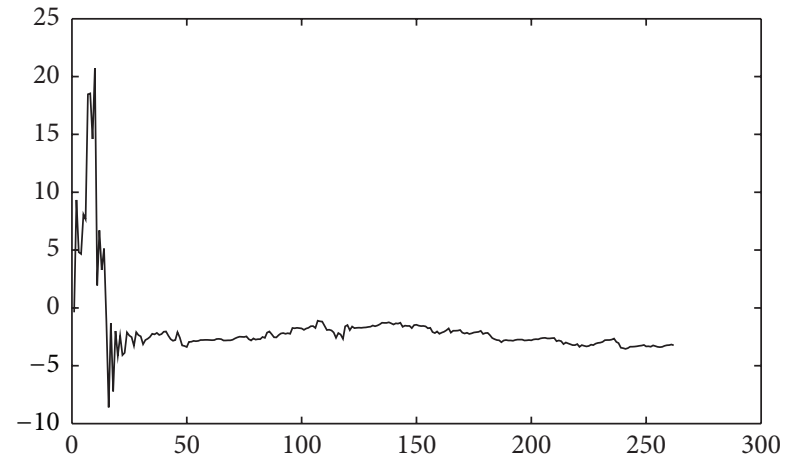

Figure 3: Values of $f(t)$ during subprime crisis.

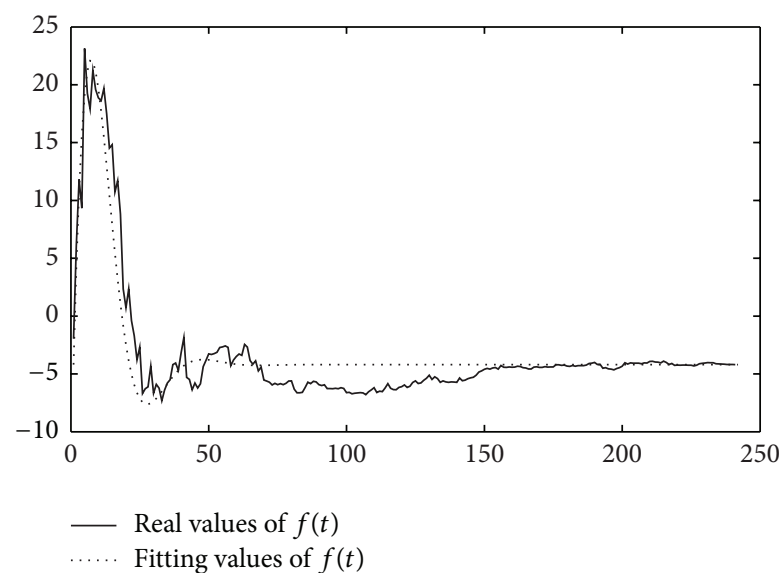

FIGURE 4: Real values versus fitting values of $f(t)$ during Asian crisis.

Given $V_{t}$, the method of estimating $(\theta, \delta)$ is to minimize the sum of squares of deviations between $\ln V_{t}$ and $\ln \sigma_{t}^{2}$; that is,

$$
\min _{(\theta, \delta)} \sum_{t=1}^{n}\left(\ln V_{t}-\ln \left(\delta^{2} S_{t}^{\theta}\right)\right)^{2}
$$

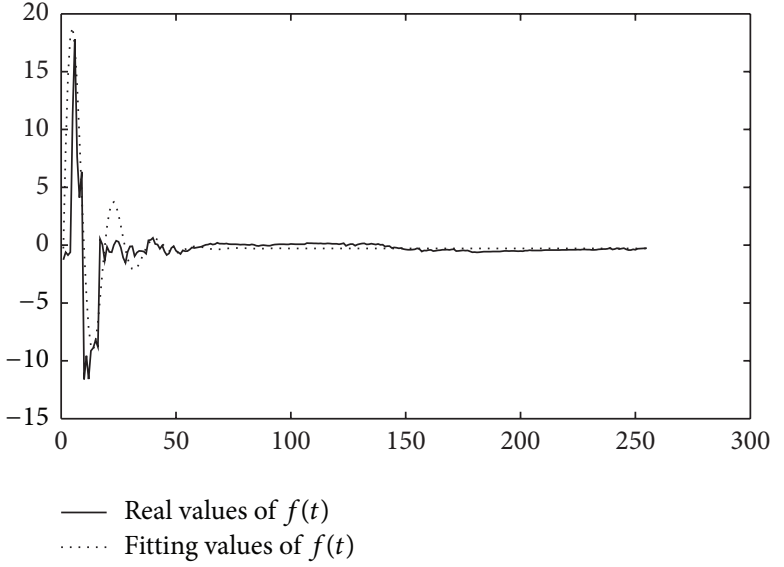

FIGURE 5: Real values versus fitting values of $f(t)$ during "9/11" event.

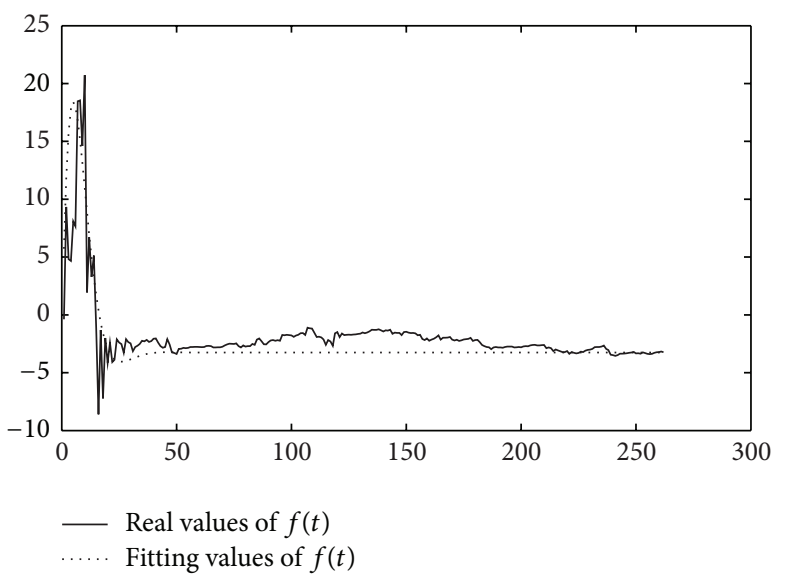

FIGURE 6: Real values versus fitting values of $f(t)$ during subprime crisis.

where $n$ is the number of data pieces. By using Newton method, we can obtain the values of $\widehat{\theta}$ and $\widehat{\delta}$ in different samples which are also exhibited in Table 1. 
4.3. Empirical Parameter Estimation of BS Model. In classical Black-Scholes model, the stock prices follow log-normal distribution. So, by using maximum likelihood estimation on different samples, the values of $\mu$ and $\sigma$ in BS model can be estimated and results are also listed in Table 1.

\section{Empirical Option Pricing Results and Analysis}

For the formula of European option pricing based on CEV model, it has been studied in many papers and we attach them in the appendix. Here, we just discuss the pricing scheme of DEV model on the European call option.

5.1. Simulation Method. Crank-Nicolson method is an efficient and widely used numerical method due to its great property, unconditionally stable. So, in this paper, we employ this method to calculate numerical solution of (8). Let $\Delta S$ and $\Delta t$ denote the sizes of the space step and time step, respectively, and then by Crank-Nicolson method we have the discrete equation as follows:

$$
\begin{aligned}
& \frac{C_{j}^{i+1}-C_{j}^{i}}{\Delta t}-\frac{r}{2}\left(C_{j}^{i+1}+C_{j}^{i}\right) \\
& +\frac{r(j \Delta S)}{2}\left(\frac{C_{j+1}^{i+1}-C_{j-1}^{i+1}}{2 \Delta S}+\frac{C_{j+1}^{i}-C_{j-1}^{i}}{2 \Delta S}\right)+\frac{1}{2} \\
& \cdot \delta^{2}(j \Delta S)^{\left(B_{j}\right)} \\
& \cdot\left(\frac{C_{j+1}^{i+1}-2 C_{j}^{i+1}+C_{j-1}^{i+1}}{(\Delta S)^{2}}+\frac{C_{j+1}^{i}-2 C_{j}^{i}+C_{j-1}^{i}}{(\Delta S)^{2}}\right) \\
& =0
\end{aligned}
$$

where

$$
B_{j}=a+A e^{b(j \Delta t)} \sin (\omega j \Delta t)
$$

By simplification, we obtain

$$
a_{j} C_{j-1}^{i}+b_{j} C_{j}^{i}+c_{j} C_{j+1}^{i}=-\alpha_{j} C_{j-1}^{i+1}+\beta_{j} C_{j}^{i+1}+\gamma_{j} C_{j+1}^{i+1},
$$

where

$$
\begin{aligned}
& a_{j}=-\alpha_{j}=-\frac{r j}{4}+\frac{\delta^{2}}{4} \frac{(j \Delta S)^{B_{j}}}{\Delta S^{2}}, \\
& b_{j}=-\beta_{j}=-\frac{1}{\Delta t}-\frac{\delta^{2}}{2} \frac{(j \Delta S)^{B_{j}}}{\Delta S^{2}}-\frac{r}{2}, \\
& c_{j}=-\gamma_{j}=-\frac{r j}{4}+\frac{\delta^{2}}{4} \frac{(j \Delta S)^{B_{j}}}{\Delta S^{2}}, \\
& \beta_{j}=-\frac{1}{\Delta t}+\frac{\delta^{2}}{2} \frac{(j \Delta S)^{B_{j}}}{\Delta S^{2}}-\frac{r}{2} .
\end{aligned}
$$

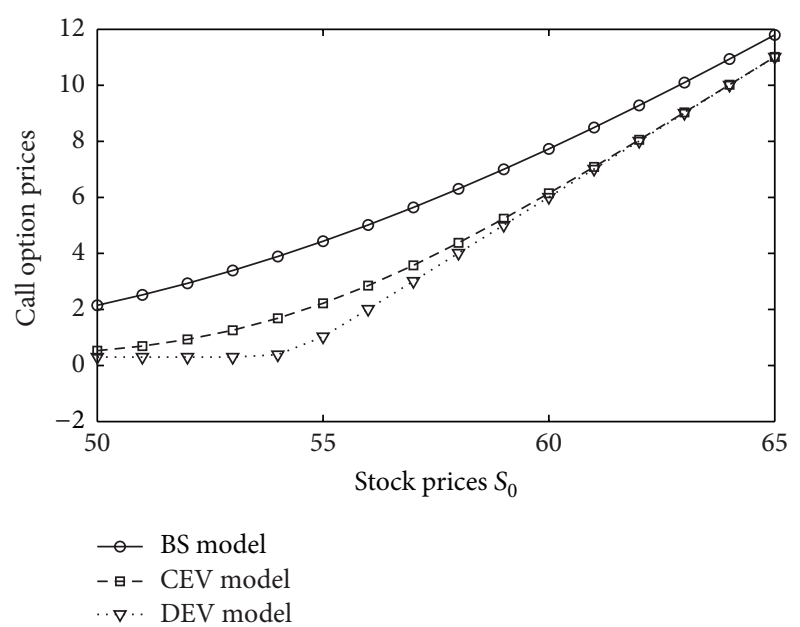

Figure 7: Call option prices at $K=53$ during Asian crisis.

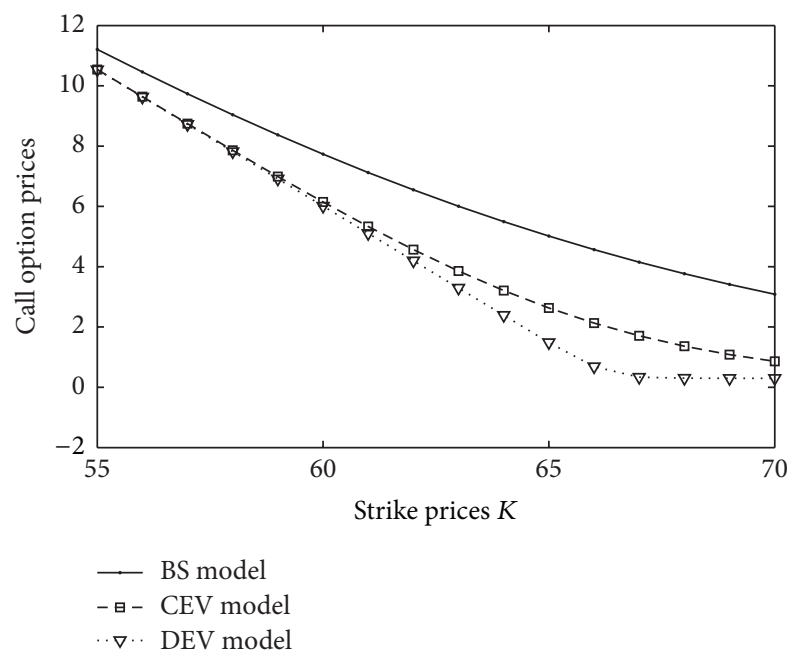

FIgURE 8: Call option prices at $S_{0}=65$ during Asian crisis.

Moreover, the boundary conditions are given as follows:

$$
\begin{aligned}
V_{0}^{i} & =0, \\
V_{j}^{N} & =\max \left(S_{j}-K, 0\right), \quad j=1,2, \ldots, M-1, \\
V_{M}^{i} & =S_{M}-K e^{-r i \Delta t}, \quad i=1,2, \ldots, N-1 .
\end{aligned}
$$

If we take risk-free interest rate $r=0.01$ and the maturity $T=1$, then the European call option prices based on the DEV model are displayed in Figures 7-12. Correspondingly, we also plot the European call option prices that are calculated by the BS model and CEV model on the same samples. In addition, in Figures 7, 9, and 11, strike price we choose is a fixed number with $K=53$ and the initial stock prices are variables, nearby this strike price. In Figures 8, 10, and 12, the initial stock prices are fixed at $S_{0}=65$, and the strike prices are variables whose ranges are selected as covering the initial stock prices. In fact, we also do some other empirical tests at different fixed strike prices and fixed initial stock prices, respectively. Their results are very similar to those given in 


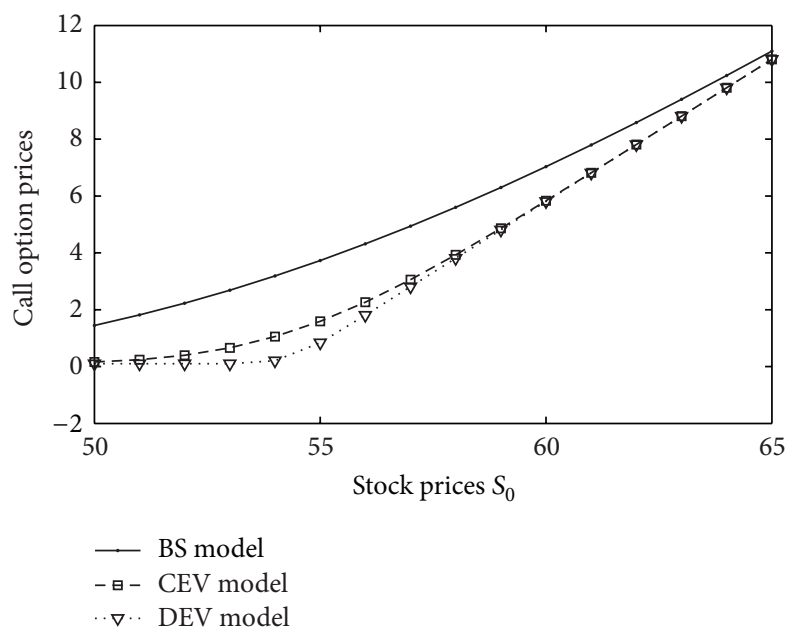

Figure 9: Call option prices at $K=53$ during "9/11" event.

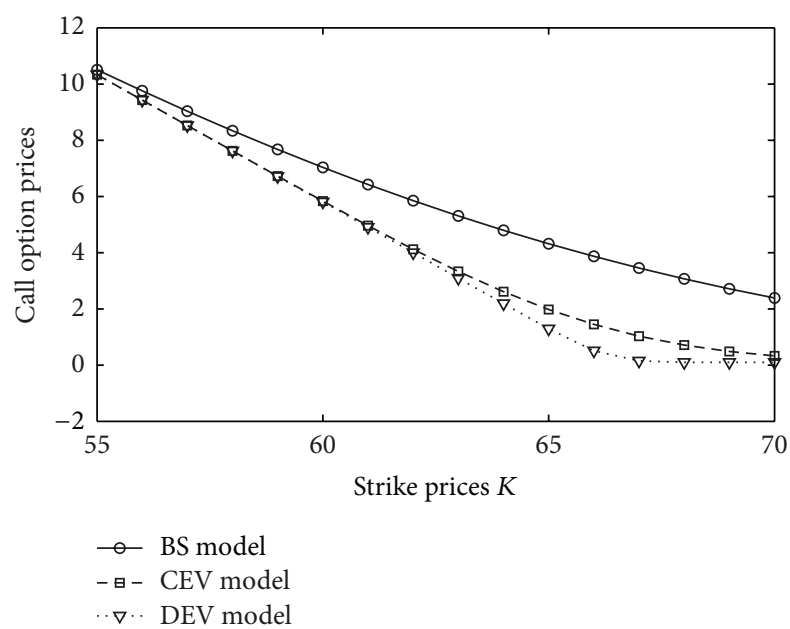

FIGURE 10: Call option prices at $S_{0}=65$ during "9/11" event.

Figures 7-12. So, we do not list out them in detail to avoid repetition.

From Figures 7-12, we obtain some important findings. The first significant phenomenon is that for all of our samples the highest line of option prices is estimated by BS model and the lowest line is achieved by DEV model. Another important finding is that, at ten percent intervals of fixed strike price and fixed initial stock price, the differences between two lines obtained by DEV model and CEV model are obvious and much bigger than the parts deep in "in-themoney" and "out-of-the-money." In addition, in the areas far from the point of "at-the-money," the performances of DEV model and CEV model are very similar, and results are almost the same.

5.2. Comparing the Errors of Option Pricing Models. In this subsection, we compare BS, CEV, and DEV models by their mean squared errors (MSE) between estimated European call

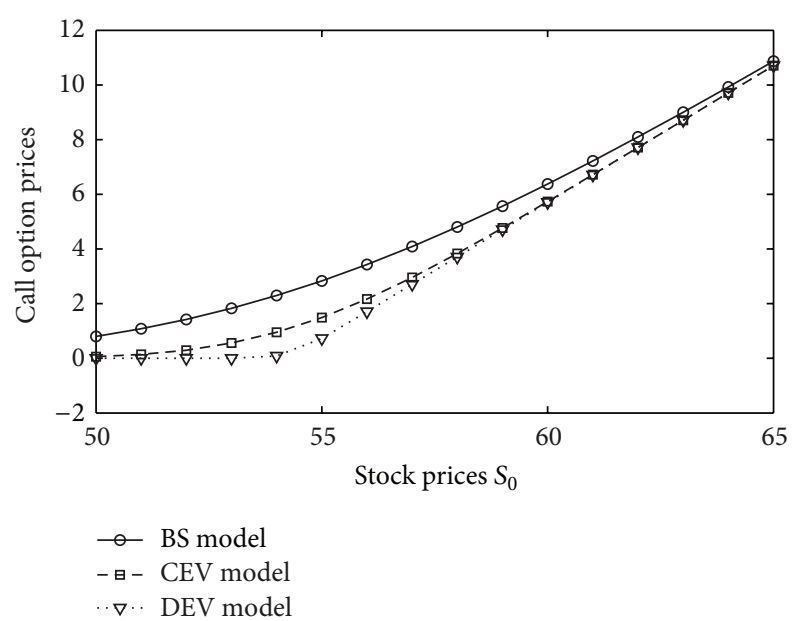

FIgURE 11: Call option prices at $K=53$ during subprime crisis.

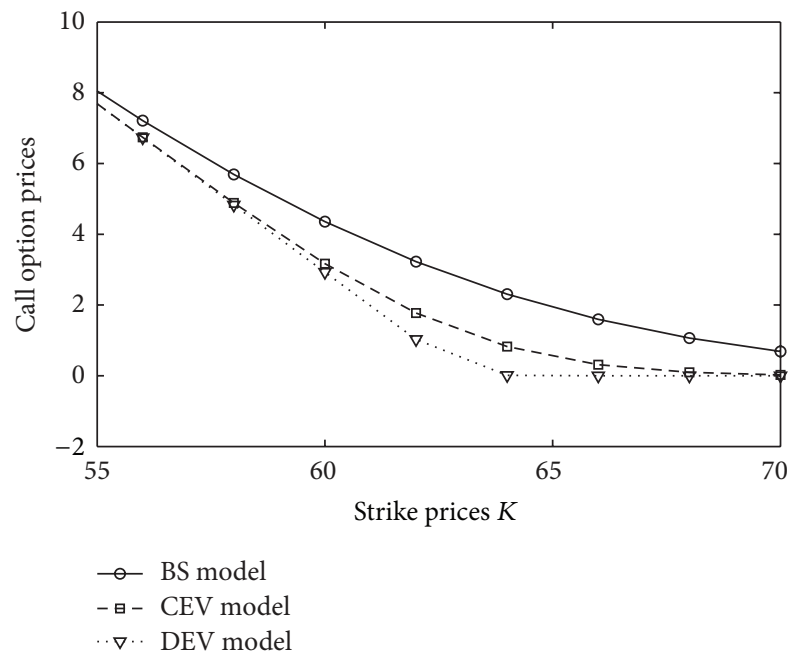

Figure 12: Call option prices at $S_{0}=65$ during subprime crisis.

option values and real option prices. The formula of MSE is given as

$$
\mathrm{MSE}=\frac{1}{N} \sum_{i=1}^{N}\left(\widehat{C}\left(K_{i}\right)-C\left(K_{i}\right)\right)^{2},
$$

where $C\left(K_{i}\right)$ denotes observed option pricing values with strike prices $K_{i}$ and $\widehat{C}\left(K_{i}\right)$ denotes estimated option pricing values by these three models. Then the implementation of the algorithm goes through the following steps.

Step 1. Take samples of stock prices and their option prices.

Step 2. Estimate parameters of BS, CEV, and DEV models, respectively.

Step 3. Calculate option prices and denote results as $\widehat{C}^{\mathrm{BS}}\left(K_{i}\right)$, $\widehat{C}^{\mathrm{CEV}}\left(K_{i}\right)$, and $\widehat{C}^{\mathrm{DEV}}\left(K_{i}\right)$.

Step 4. Calculate MSE by formula (24). 
TABLE 2: Summary MSE of BS, CEV, and DEV models.

\begin{tabular}{lccc}
\hline Sample & \multicolumn{3}{c}{ MSE } \\
& BS & CEV & DEV \\
\hline Oct. 17, 1997-Jun. 16, 1998 & 5.7601 & 4.9712 & 2.3254 \\
Sep. 10, 2001-Jun. 15, 2002 & 4.4693 & 4.0320 & 2.0185 \\
Sep. 16, 2008-Jun. 30, 2009 & 4.9876 & 4.1724 & 2.6968 \\
\hline
\end{tabular}

By simulation, the MSE of these three models in different samples are exhibited in Table 2.

So, from results above, we can see that for different samples the MSE of DEV model is the smallest one of these three models, and the MSE of CEV model is smaller than that of BS model. Therefore, the DEV model we proposed has the best performance in option pricing estimations.

\section{Conclusion}

In this paper, our main contribution is to propose a fitted and effective model, DEV model, to fit stock prices and to estimate option prices in the market with financial crisis by considering that the elasticity of variance is dynamic. Based on this model, European option pricing problem has been analyzed and the partial differential equation for option prices has been derived. In addition, we provide efficient estimated methods, Kalman filtering method and least square method, for the parameters in the volatility term. Moreover, Crank-Nicolson method has been implemented to obtain the numerical solution of the option prices. The MSE of empirical results shows that DEV model is more accurate than CEV model and BS model to fit the real option prices.

Considering the fact that there are lots of jumps and rebounding cases in crashes, it may affect the prices of path dependent options. So, in the future, we will focus on how to grasp the jump events in the financial crisis by improving DEV model to estimate American options and Asian options.

\section{Appendix}

\section{CEV Call Option Pricing Formula}

In [23], the formula of CEV model on European call option is given as follows:

$$
C\left(t, S_{t}\right)=S_{t} M_{1}-K e^{-r(T-t)} M_{2}
$$

in which $M_{1}$ and $M_{2}$ are given by

$$
\begin{aligned}
& M_{1} \\
& = \begin{cases}\sum_{n=0}^{\infty} g\left(S_{1} \mid n+1\right) \cdot G\left(K_{1} \mid n+p\right), & \theta>2 \\
1-\sum_{n=0}^{\infty} g\left(S_{1} \mid n+p\right) \cdot G\left(K_{1} \mid n+1\right), & \theta<2\end{cases}
\end{aligned}
$$

$$
\begin{aligned}
& M_{2} \\
& \quad= \begin{cases}\sum_{n=0}^{\infty} g\left(S_{1} \mid n+p\right) \cdot G\left(K_{1} \mid n+1\right), & \theta>2 \\
1-\sum_{n=0}^{\infty} g\left(S_{1} \mid n+1\right) \cdot G\left(K_{1} \mid n+p\right), & \theta<2,\end{cases}
\end{aligned}
$$

where

$$
\begin{aligned}
& S_{1}=\frac{2 r \cdot \exp [r(T-t)(2-\theta)] S^{2-\theta}}{\delta^{2}(2-\theta) \cdot \exp [r(T-t)(2-\theta)]-\delta^{2}(2-\theta)}, \\
& K_{1}=\frac{2 r K^{2-\theta}}{\delta^{2}(2-\theta) \cdot \exp [r(T-t)(2-\theta)]-\delta^{2}(2-\theta)}, \\
& g(x \mid m)=\frac{e^{-x} x^{m-1}}{\Gamma(m)}, \\
& G(x \mid m)=\int_{x}^{\infty} g(y \mid m) d y \\
& p=1+\frac{1}{|2-\theta|} .
\end{aligned}
$$

\section{Competing Interests}

The authors declare that they have no competing interests.

\section{Acknowledgments}

The authors are especially grateful for anonymous referees for comments and suggestions that greatly improved this paper. The work on this paper was partially supported by the Fundamental Research Funds for the Central Universities (nos. JBK1507113, JBK150931, and JBK160207).

\section{References}

[1] F. Black and M. Scholes, "The pricing of options and corporate liabilities," Journal of Political Economy, vol. 81, no. 3, pp. 637654, 1973.

[2] R. C. Blattberg and N. J. Gonedes, "A comparison of the stable and student distributions as statistical models for stock prices," The Journal of Business, vol. 47, no. 2, pp. 244-280, 1974.

[3] J. Cox, "Note on option pricing 1: constant elasticity of diffusion,” Draft, Stanford University, Stanford, Calif, USA, 1976.

[4] J. C. Cox and S. A. Ross, "The valuation of options for alternative stochastic processes," Journal of Financial Economics, vol. 3, no. 1-2, pp. 145-166, 1976.

[5] D. Jong and R. Huismn, "From skews to a skewed-t: modelling option-implied returns by a skewed student-t," in Proceedings of the IEEE/IAFE/INFORMS Conference on Computational Intelligence for Financial Engineering (CIFEr'00), New York, NY, USA, March 2000.

[6] S. Markose and A. Alentorn, "The generalized extreme value distribution, implied tail index, and option pricing," The Journal of Derivatives, vol. 18, no. 3, pp. 35-60, 2011.

[7] D. Zhu and J. Galbraith, "A generalized asymmetric student's t distribution with applications to financial economics," Journal of Economics, vol. 157, no. 6, pp. 297-305, 2010. 
[8] D. C. Emanuel and J. D. MacBeth, "Further results on the constant elasticity of variance call option pricing model," The Journal of Financial and Quantitative Analysis, vol. 17, no. 4, pp. 533$554,1982$.

[9] T. Nawdha, T. Yannick, and B. Muddun, "Efficient and high accuracy pricing of barrier options under the CEV diffusion," Journal of Computational and Applied mathematics, vol. 280, pp. 182-193, 2015.

[10] T. J. Finucane, "Black-Scholes approximations of call option prices with stochastic volatilities: a note," Journal of Financial and Quantitative Analysis, vol. 24, no. 4, pp. 527-532, 1989.

[11] C. Wang, S. W. Zhou, and J. Y. Yang, "The pricing of vulnerable options in a fractional brownian motion environment," Discrete Dynamics in Nature and Society, vol. 2015, Article ID 579213, 10 pages, 2015.

[12] J. Huang, W. Zhu, and X. Ruan, "Option pricing using the fast Fourier transform under the double exponential jump model with stochastic volatility and stochastic intensity," Journal of Computational and Applied Mathematics, vol. 263, pp. 152-159, 2014.

[13] X. S. Yu and L. Yang, "Pricing american options using a nonparametric entropy approach," Discrete Dynamics in Nature and Society, vol. 2014, Article ID 369795, 16 pages, 2014.

[14] Y. Xing and X. Yang, "Equilibrium valuation of currency options under a jump-diffusion model with stochastic volatility," Journal of Computational and Applied Mathematics, vol. 280, pp. 231247, 2015.

[15] F. Black, "Fact and fantasy in the use of option," Financial Analysts Journal, vol. 31, no. 4, pp. 36-41, 1975.

[16] E. Platen and R. Sidorowicz, "Empirical evidence on Student $\mathrm{t}$ log-returns of diversified world stock indices," Quantitative Finance Research Center, vol. 2, no. 2, pp. 132-142, 2000.

[17] G. Dibeh and H. M. Harmanani, "Option pricing during postcrash relaxation times," Physica A: Statistical Mechanics and its Applications, vol. 380, no. 1-2, pp. 357-365, 2007.

[18] F. Lillo and R. Mantenga, "Power-law relaxation in a complex system: omori law after a financial market crash," Rosario Mantegna, vol. 68, no. 1, pp. 65-70, 2003.

[19] D. Sornette, Why Stock Markets Crash: Critical Events in Complex Financial Markets, Princeton University Press, Princeton, NJ, USA, 2003.

[20] Y. El-Khatib, M. A. Hajji, and M. Al-Refai, "Options pricing in jump diffusion markets during financial crisis," Applied Mathematics and Information Sciences, vol. 7, no. 6, pp. 2319-2326, 2013.

[21] J.-H. Yoon, "Pricing perpetual American options under multiscale stochastic elasticity of variance," Chaos, Solitons \& Fractals, vol. 70, no. 1, pp. 14-26, 2015.

[22] J.-H. Yoon and C.-R. Park, "Pricing turbo warrants under stochastic elasticity of variance," Chaos, Solitons \& Fractals, 2015.

[23] M. Chesney, R. Ellott, D. Madan, and H. Yang, "Diffusion coefficient estimation and asset pricing when risk premia and sensitivities are time varying," Mathematical Finance, vol. 3, no. 2, pp. 85-99, 1993. 


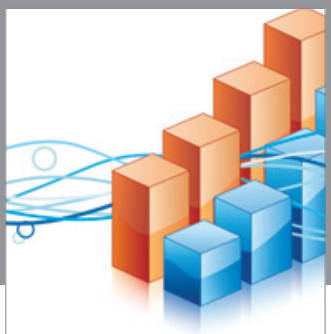

Advances in

Operations Research

vatem alat4

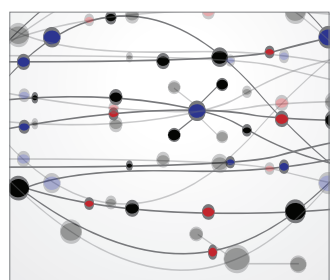

\section{The Scientific} World Journal
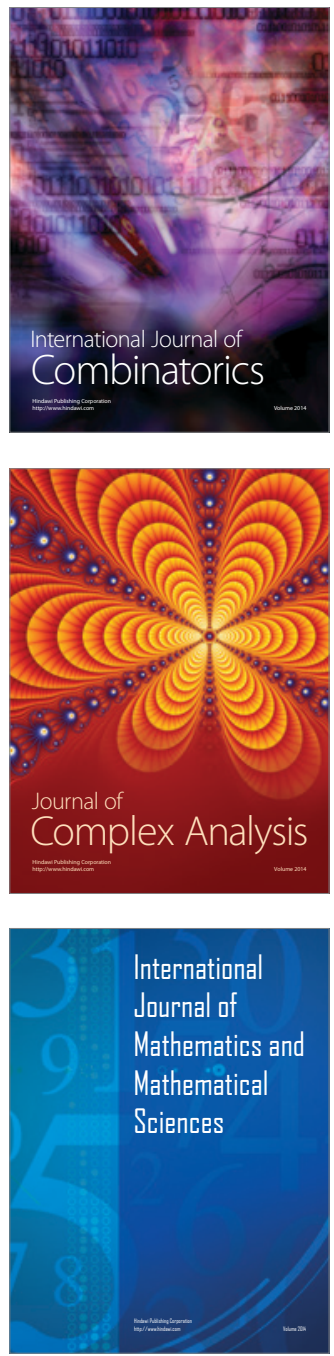
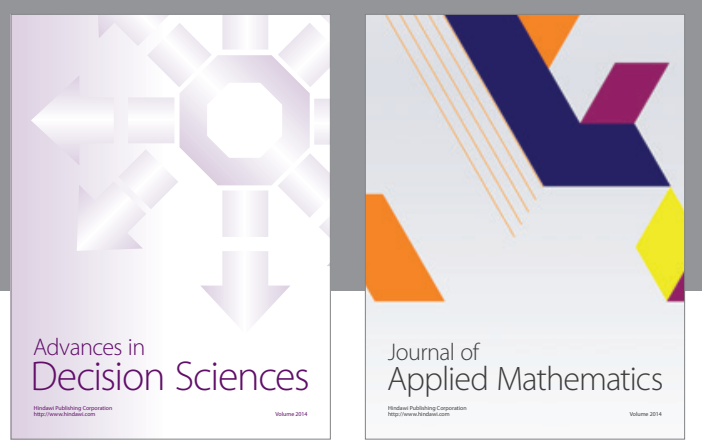

Algebra

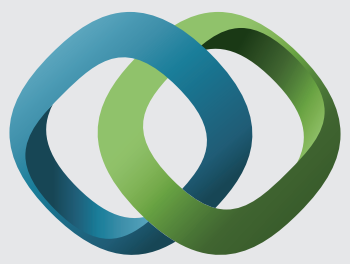

\section{Hindawi}

Submit your manuscripts at

http://www.hindawi.com
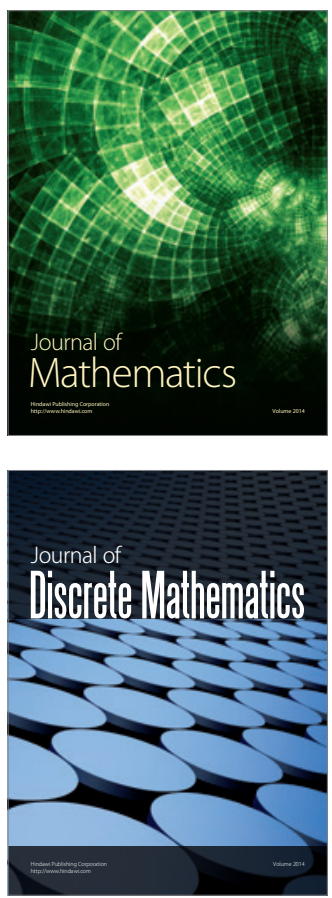

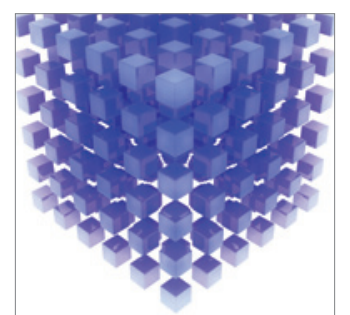

Mathematical Problems in Engineering
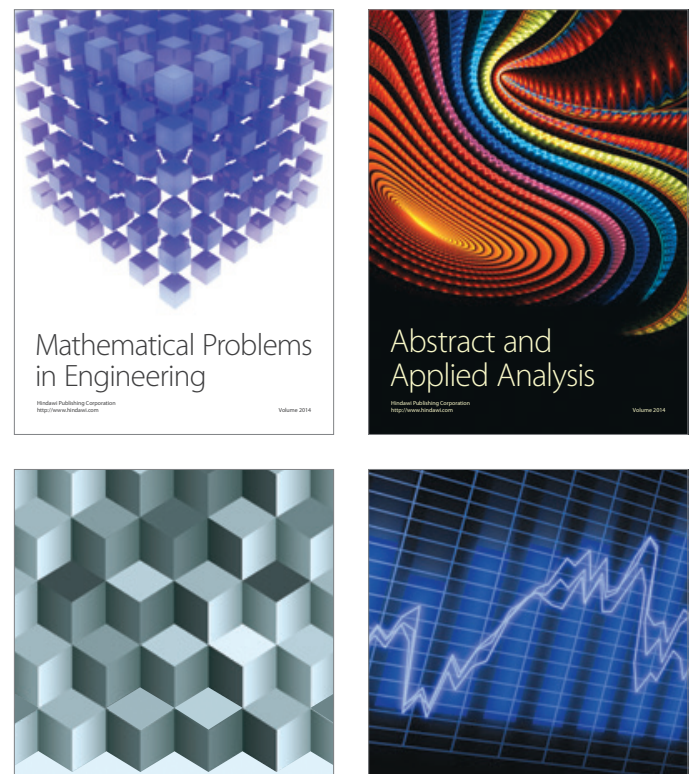

Journal of

Function Spaces

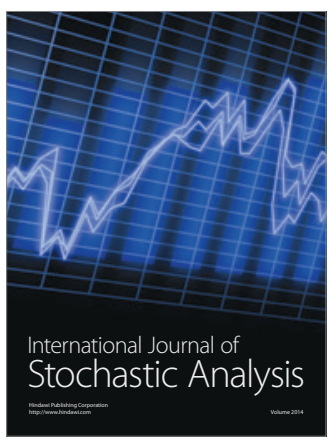

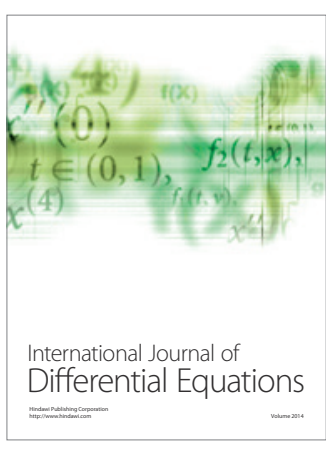
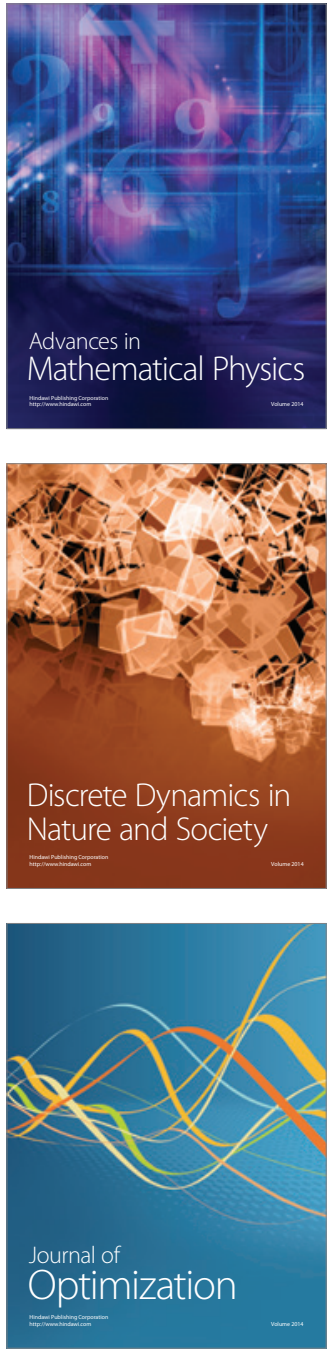